\title{
Surgical Treatment of Malignant Peritoneal Mesothelioma: Past, Present, and Future
}

\author{
H. Richard Alexander Jr., MD \\ Department of Surgery, University of Maryland, School of Medicine, Baltimore, MD
}

Malignant peritoneal mesothelioma (MPM) is a rare malignancy that arises from the serosal membranes of the abdominal cavity. In 1972, Moertel published a paper characterizing the clinical course of patients afflicted with MPM and the now recognized clinical features associated with tumor progression within the abdominal cavity. ${ }^{1}$ Borow first reported the association between asbestos exposure and mesothelioma that same year. ${ }^{2}$ Despite the current recognition that MPM is a distinct entity from its pleural variant, progress in the treatment of patients afflicted with MPM has been hampered by several factors. First, it is an extraordinarily rare condition: only $10-15 \%$ of all patients with mesothelioma present with the peritoneal form of the disease, and this translates into approximately 300-400 cases in the USA annually. As a consequence, throughout the last two decades many clinical trials testing systemic agents for patients with malignant mesothelioma did not distinguish between those with the pleural versus the peritoneal form of disease. ${ }^{3-5}$ Many of these trials included a small number of patients with MPM and for whom no confident conclusions regarding efficacy of the experimental regimen could be made. It is now becoming increasingly recognized that patients with MPM have a distinct disease for which specific therapies should be developed.

Another significant feature of MPM that has challenged our ability to understand the contribution of various therapeutic interventions to patient outcome is the remarkable variability in the biology of this condition. In 1973, Rogoff and colleagues reported outcomes in four patients treated at Memorial Sloan-Kettering Cancer Center in New York using surgical cytoreduction and whole-abdominal

(C) Society of Surgical Oncology 2009

Published Online: 4 November 2009

H. R. Alexander Jr., MD

e-mail: HRAlexander@smail.umaryland.edu radiotherapy. ${ }^{6}$ Two of the four patients survived longer than 5 years. Antman and colleagues reported outcomes of 37 patients with MPM treated at the Dana Farber Cancer Institute and the Brigham and Women's Hospital between 1965 and 1984 and also noted that, while some patients progressed and succumbed rapidly, others survived for many years after treatment. ${ }^{7}$ In their series, patients treated after 1982 underwent cytoreduction, placement of an intraperitoneal (IP) catheter, and received between 8-10 cycles of IP doxorubicin and cisplatin. Subsequent to that $30 \mathrm{~Gy}$ of whole-abdominal radiation was administered. In their analysis, patients with smaller tumor burden and female gender had prolonged survival; those with epithelioid tumors did poorly compared with those with other histopathological types. Since that initial description, the use of multimodal therapy using cytoreduction with some form of high-dose regional chemotherapy has become increasingly utilized.

Because MPM progresses almost exclusively in the abdominal cavity, locoregional therapies designed to control disease progression in the abdomen appear justified. Cytoreduction and hyperthermic intraoperative intraperitoneal perfusion with chemotherapy (HIPEC) takes advantage of two complementary treatments. Cytoreduction has the goal of achieving a complete resection of all grossly identifiable tumor, and high-dose chemotherapy is intended to treat the micrometastatic residual sites of disease. The use of HIPEC has the theoretical advantage of being the most effective way of uniformly distributing high-dose IP chemotherapy to all of the peritoneal surfaces at risk of harboring disease and allows one to apply chemotherapy with clinically relevant amounts of hyperthermia, which is known to enhance the cytotoxic effects of multiple different types of chemotherapeutic agents. Outcomes using this strategy in selected patients with a disease burden amenable to resection and with a good performance status have been the topic of numerous reports. They show that patient 
outcome after this therapy is variable and that treatment is associated with some morbidity and occasional mortality. ${ }^{8}$ Therefore, identification of prognostic factors that could aid in patient selection and treatment planning are of increasing importance.

In this issue, Dr. Baratti and colleagues analyze the significance of lymph node metastases as a possible independent prognostic factor associated with outcome in patients undergoing cytoreduction and HIPEC for MPM. ${ }^{9}$ Their data suggest that the overall frequency of lymph node metastases is low; in a group of 15 patients whose nodes were routinely sampled the overall frequency of nodal metastases was $20 \%$, compared with $40 \%$ in a cohort whose lymph nodes appeared to be pathological enlarged and suspicious. In the overall series of 83 patients, they identified epithelioid histology, low mitotic count, complete gross cytoreduction, and pathologically negative nodes as independent factors associated with increased survival. It is not clear from the paper whether or not the lymph node metastases were a consequence of advanced tumor burden versus tumor biology. There was a notable association between a higher peritoneal cancer index, a reflection of tumor burden within the peritoneal cavity, and node positivity, suggesting that nodal metastases may simply have been the consequence of extensive tumor burden within the peritoneal tissues. It is noteworthy that no patient with lymph node metastases developed recurrences in nodal basins on follow-up.

The challenge for us now is to understand how to use this information to assist us in the management of patients with MPM. There are a number of factors that have been consistently recognized as important in predicting better outcome in patients undergoing cytoreduction and HIPEC such as age, histology, and the ability to achieve a complete gross cytoreduction. Whether or not nodal status can now be used routinely to predict better outcome in cases of pathological negative nodes or indicate a high risk of early recurrence and disease progression in cases of pathological positive nodes needs to be validated in additional studies. Ultimately, the understanding of this disease's clinical behavior may best be determined by a better understanding of its molecular biology.

The authors are from the National Cancer Institute of Italy and are acknowledged leaders in the treatment of MPM. It is remarkable that, at a world-recognized center of expertise in this area, the number of patients reported with diffuse MPM over a 13-year interval was only 83. Their volume of patients with this condition compares similarly to other centers around the world with acknowledged expertise in treating this condition. For us to make meaningful advances in the treatment of this condition we must now move from reporting institutional experiences to more collective and cooperative efforts in the study of this rare disease.

\section{REFERENCES}

1. Moertel CG. Peritoneal mesothelioma. Gastroenterology. 1972;63: 346-50.

2. Borow M, Conston A, Livornese LL, et al. Mesothelioma and its association with asbestosis. JAMA. 1967;201:587-91.

3. Samson MK, Wasser LP, Borden EC, et al. Randomized comparison of cyclophosphamide, imidazole carboxamide, and adriamycin versus cyclophosphamide and adriamycin in patients with advanced stage malignant mesothelioma: a Sarcoma Intergroup Study. J Clin Oncol. 1987;5:86-91.

4. Chahinian AP, Antman K, Goutsou M, et al. Randomized phase II trial of cisplatin with mitomycin or doxorubicin for malignant mesothelioma by the Cancer and Leukemia Group B. J Clin Oncol. 1993;11:1559-65.

5. Govindan R, Kratzke RA, Herndon JE, et al. Gefitinib in patients with malignant mesothelioma: a phase II study by the Cancer and Leukemia Group B. Clin Cancer Res. 2005;11:2300-4.

6. Hassan R, Remaley AT, Sampson ML, et al. Detection and quantitation of serum mesothelin, a tumor marker for patients with mesothelioma and ovarian cancer. Clin Cancer Res. 2006;12:447-53.

7. Antman K, Osteen R, Klegar K, et al. Early peritoneal mesothelioma: a treatable malignancy. Lancet. 1985;2:977-81.

8. Alexander HR, Hanna N, Pingpank JF. Clinical results of cytoreduction and HIPEC for malignant peritoneal mesothelioma. Cancer Treat Res. 2007;134:343-55.

9. Baratti D, Kusamura S, Cabras AD, Laterza B, Balestra MR, Deraco M. Lymph-node metastases in diffuse malignant peritoneal mesothelioma. Ann Surg Oncol. 2009. 